\title{
Aus der Krise lernen? Über neue Abläufe und Führungsqualitäten, die sich bewähren
}

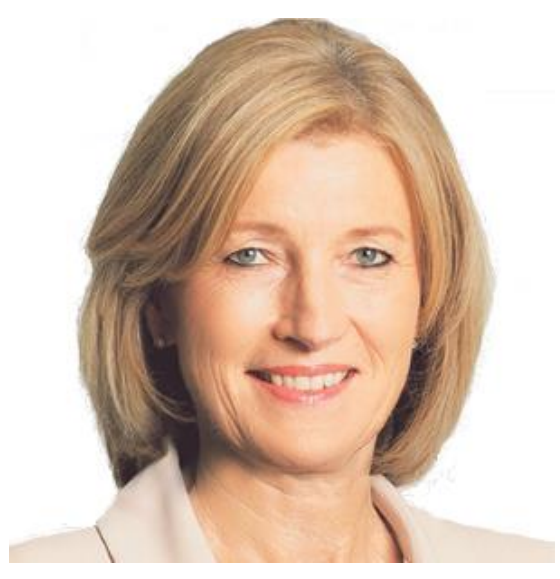

Foto: Martina Salomon, zVg (Kurier)
Ihre Karriere begann die mehrfach ausgezeichnete österreichische Journalistin Dr. Martina Salomon, promovierte Germanistin und Publizistin zunächst als freie Mitarbeiterin im ORF-Landesstudio Oberösterreich sowie bei den Oberösterreichischen Nachrichten. In der Folge war sie für die Tiroler Tageszeitung und den Standard tätig. Ab 2004 leitete Salomon das Innenpolitik-Ressort der Presse. Ihre wöchentliche Kolumne "Salomonisch - Das innenpolitische Wort zum Sonntag" erschien zunächst in der Presse am Sonntag und seit Oktober 2010 jeden Samstag in der Tageszeitung Kurier. Als Chefredakteurin setzt sie seit 2018 mitunter auch auf die Expertise der Praktiker*innen: Sie gründete den "Kurier-Bildungsbeirat", in welchen ich mit Vertreter*innen aller Schularten berufen wurde. Seither kennen wir uns persönlich und so kam das Interview zustande, in Zeiten wie diesen auf der Mailschiene.

Seit einem Jahr hält uns die COVID-19-Pandemie in Atem - mit Auswirkungen auf viele Lebensbereiche. Welche neuen Herausforderungen hat die Krise an Sie als Führungskraft in einem Medienunternehmen, als Chefredakteurin des "Kurier" gestellt?

Martina Salomon: Einen wesentlichen Unterschied stellt dar, dass wir nur mit zwanzig Prozent der Mannschaft im Newsroom sitzen. Von dort werden ja neben der Zeitung auch noch kurier.at, Schau-TV, der Kurier-Podcast und der Newsletter gesteuert. Wir sind in zwei Teams geteilt, die wegen der Pandemie wochenweise wechseln. Bisher hat das gut geklappt, das Büro wurde in Windeseile technisch aufgerüstet.

Wie würden Sie Ihren Führungsstil beschreiben? Und welche Eigenschaften halten Sie im Moment für besonders wichtig, um ein großes Team und das Unternehmen gut durch die Krise zu führen?

Ich pflege einen kollegialen Stil, sitze auch selbst mitten im Großraumbüro, bin also sehr „nahbar". In der Krise ist es, denke ich, besonders wichtig, für alle ein offenes Ohr zu haben, um Überforderung beziehungsweise manchmal auch Unterforderung rechtzeitig zu erkennen und darauf reagieren zu können. 


\section{Aus der Krise „geborene“ Neuerungen}

Auch wenn das Ende der Pandemie noch nicht in Sicht ist: Wenn ich mit Schulleiter*innen in ganz Österreich spreche, sind wir uns einig, dass manche Begleiterscheinungen der Krise bleiben werden, weil sie sich bewährt haben. In unserem Bereich sind das die Digitalisierung generell, sei es in den organisatorischen Abläufen oder in der direkten Kommunikation mit allen Schulpartner*innen, sei es der Einsatz digitaler Medien im Unterricht. Wir sind auch froh über den Zusammenhalt in unseren Teams, die die meisten von uns als große Stütze empfinden. Gibt es in Ihrem Bereich Dinge, die sich in der Krise bewährt haben und die Sie beibehalten werden?

Die Möglichkeit, sich von „außen“ digital an unserer analogen Konferenz im Newsroom zu beteiligen, nehmen sehr viele Kolleg*innen in Anspruch. Das wird sicher bleiben. Auch Homeoffice wird es künftig öfter geben als vor der Corona-Krise. Dennoch ist es für kreative Sitzungen nach wie vor wichtig, sich analog zu treffen. Journalismus braucht in jeder Hinsicht Begegnung. Ich bin also nicht begeistert von dauerhaften Homeoffices. Da verliert man Kontakt zu den Mitarbeitenden und zur Themenvielfalt.

Meiner Wahrnehmung nach standen Schulleiter*innen medial noch nie so im Fokus wie in dieser Zeit. Wir sind ja meist nur dann in den Medien, wenn irgendwo etwas Unerfreuliches passiert. Sie selbst haben im Kurier schon lange vor der Krise einen sogenannten "Bildungsbeirat" installiert, dem ich gemeinsam mit Vertreter*innen aller pädagogischen Bereiche angehören darf. Hat sich Ihr Bild von Schule bzw. vom Wirken der Direktor*innen dadurch geändert bzw. gibt es etwas, was Sie Direktor*innen als Medienfachfrau raten würden?

Ich habe gelernt, dass die Behörden noch längst nicht im digitalen Zeitalter angekommen sind. Das ist den Schulen besser gelungen. Das Feedback (aber mehr das persönliche, als das recherchierte) von vielen Familien auch im Kolleg*innenkreis war, dass es haushohe Unterschiede zwischen den einzelnen Lehrkräften gab - manches hat super, manches gar nicht funktioniert. Und die Familien waren zum Teil sehr überfordert und frustriert (was sicher auch auf die Lehrer*innen zutraf). Mein Bild hat sich nicht geändert, weil ich schon immer der Meinung war, dass das Lehrpersonal der Schlüssel für gelingende Schule ist und darauf noch immer zu wenig geachtet wird - im positiven (toll, was die Lehrer*innen leisten) wie im negativen Sinne (Ausbildung, Auswahl - wahrscheinlich beides sehr verbesserbar)! Und die Krise hat noch härter zutage gebracht, dass ein bildungsfernes Elternhaus für Schüler*innen im österreichischen System eine besondere Katastrophe ist, die man im späteren Leben kaum mehr gutmachen kann.

Mit Martina Salomon sprach Isabella Zins 